\section{Error detection performance in a proportion estimation task}

\author{
JOHN A. HICKS III \\ North Carolina State University, Raleigh, N.C. 27607
}

This study established some baseline data in an error detection task involving proportion estimation. Stimuli consisted of a standard random arrangement of 100 " + " signs with a variable number of + signs circled. With each stimulus presentation, Ss attempted to detect an error or difference between the actual number of + signs circled and a numerical estimate provided with the presentation. The detection data were utilized to calculate differential thresholds for error detection. Performance was superior with proportions less than $50 \%$. Ss detected negative errors, i.e., when the numerical estimate provided was less than the actual number of + signs circled, better than positive errors.

Although a great deal of perceptual research has been done in the area of thresholds, very little literature is available in the particular area of error or discrepancy detection. Error detection has been investigated in industrial settings. This research has involved topics concerning inspection accuracy and detection of out-of-tolerance conditions with different types of displays. The error detection task in the present study involved proportion estimation. With regard to proportion estimation, earlier studies by Shuford (1961) and others revealed the complex nature of a simple proportion estimation task. The responses required of $\mathrm{Ss}$ in the present study made the experimental task more complex than a proportion estimation task by itself, because in addition to estimating proportions the Ss were required to make absolute judgments regarding the presence or absence of errors.

On the basis of studies by Shuford (1961) and Stevens \& Galanter (1957), it was hypothesized in the present study that differential thresholds of error detection would vary and approximate a binomial distribution as a function of the proportions being estimated. This hypothesis would have been supported if the differential thresholds at the upper and lower ends of the proportion continuum were approximately equal and significantly smaller than the differential threshold at the middle of the continuum. A second hypothesis tested was that the direction of the error in the presentation has a significant effect upon error detection performance. A third hypothesis tested was that the size of the error in the presentation has a significant effect upon performance.

Ss viewed a number of stimulus presentations which consisted of a standard random arrangement of 100 "+" signs, with a variable number of + signs circled (see Fig. 1). With each 100 " + signs.
The apparatus consisted of an automatic Kodak Carousel Model 800 slide projector, a projection screen, a set of $6535-\mathrm{mm}$ slides, and answer sheets. All the slides contained the identical random arrangement of 100 + signs contained in Fig. 1. Varying numbers of + signs were circled on each slide. Thirty practice slides were used and 70 presentation slides were employed."

All Ss were tested in one group. Initially, they were given some practice in simple proportion estimation. During additional practice slides and during the presentation slides Ss were instructed to respond "higher" if the number in the box was larger than their proportion estimate of the background or "lower" if the number in the box was smaller.

After the practice slides, Ss were stimulus presentation, Ss attempted to detect an error or difference between the actual number of + signs circled and a numerical estimate provided with the presentation. Both the direction of the error, i.e., whether the numerical estimate was less than or greater than the actual number of + signs circled, and the size of the algebraic error, i.e., the difference between the actual number of + signs circled and the number entered in the lower right-hand corner, were varied. Ss received ample time to inspect each presentation and, although reaction time was not important, they were encouraged to respond quickly and always to estimate the number of encirled + signs rather than count them.

\section{EXPERIMENT 1}

Ten undergraduate students in introductory psychology courses at the University of Texas at El Paso served as Ss. presented a set of 35 presentation slides in five blocks in random order for a total of 175 presentations. Each slide was presented for $5 \mathrm{sec}$, and then $5 \mathrm{sec}$ were allowed for the recording of responses.

The data were employed to calculate differential thresholds using the method of constant stimulus differences, as described by Corso (1967), for five different proportions $(20,35,50,65$, and 80$)$. The data were also used to compare detection performance with these different proportions, error sizes, and error direction, employing a 5 by 2 by 3 repeated measures design with $10 \mathrm{Ss}$, with the dependent variable being the number of correct responses. This design involved 5 different proportions, 2 error directions (positive, negative), and 3 different error sizes $(5,10,15)$. The 35

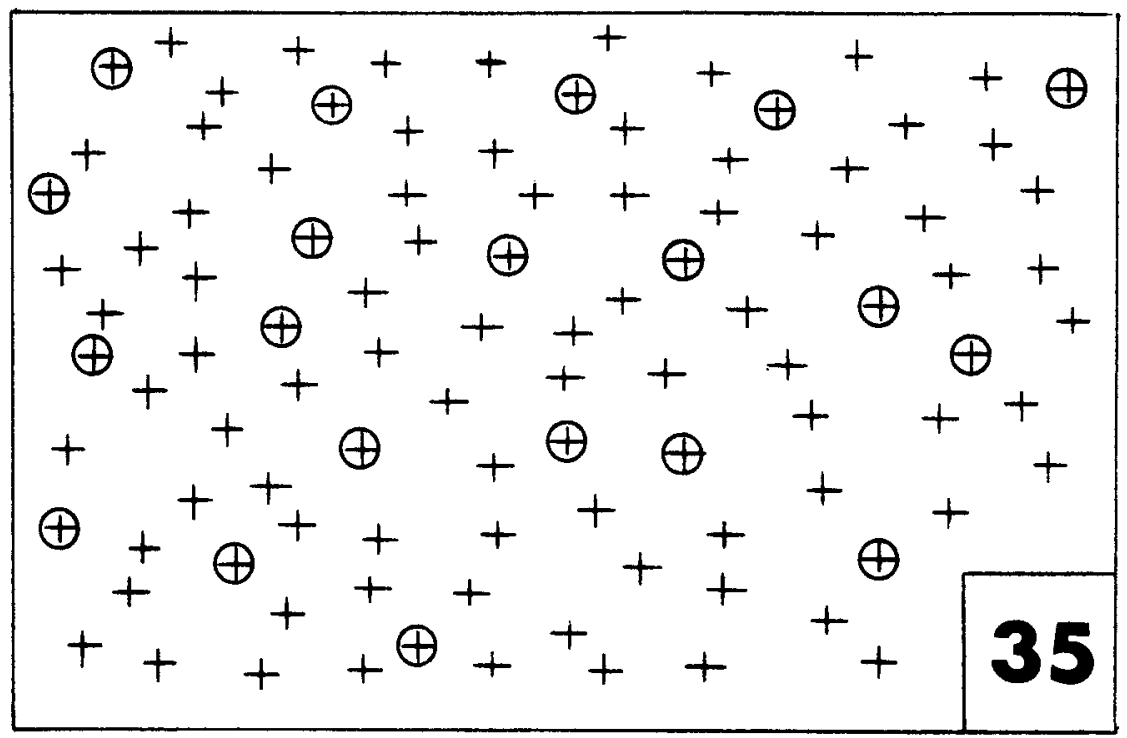

Fig. 1. Example of stimulus presentation containing random arrangement of 
Threshold Results

\begin{tabular}{crrrr}
\cline { 2 - 4 } Proportion & $\mathrm{H}_{\mathrm{DL}^{*}}$ & $\mathrm{~L}_{\mathrm{DL}}{ }^{*}$ & $\mathrm{IU}+$ & Mean DL \\
\hline 20 & 3.17 & -3.69 & 6.86 & 3.43 \\
35 & 6.78 & -2.25 & 9.03 & 4.52 \\
50 & 6.39 & -8.50 & 14.89 & 7.44 \\
65 & 10.83 & -4.50 & 15.33 & 7.66 \\
80 & 7.90 & -6.04 & 13.94 & 6.97 \\
Mean & 7.01 & -5.00 & 12.01 & 6.00 \\
\hline
\end{tabular}

*Upper differential threshold **Lower differential threshold $\quad$ Interval of uncertainty

presentation slides were generated by the combinations of 5 proportions, 2 error directions, and the 3 error sizes for a total of 30 slides plus 1 slide for each of the 5 proportions with no error present.

\section{Results}

The calculated differential thresholds were not in agreement with the experimental hypothesis, i.e., no binomial distribution of differential thresholds for detection performance was observed (see Table 1). It was found that the proportion factor differentially affected detection performance, $F(4,36)=3.70$, $p<.025$. Also, error size significantly affected performance, $F(2,18)=$ 76.92, $\mathrm{p}<.001$. A Duncan's multiple-range test $(p<.01)$ indicated that $\mathrm{Ss}$ performed significantly better with the 20 proportion than with the 35 proportion. Ss' performance with both of these proportions was significantly better than with the 50 , 65 , and 80 proportions; $\mathrm{Ss}^{\prime}$ performances with these three proportions were not significantly different. A Duncan's test $(p<.01)$ also indicated that $S s$ performed significantly better with the 15 and 10 error sizes than with the 5 error size, although there was no significant difference in Ss' performances between the 15 and 10 error sizes. The Proportion by Error Size interaction was significant, $\mathbf{F}(8,72)=21.10$, $\mathrm{p}<.001$. This was primarily due to the variability in performance obtained with the 5 error size. The Proportion by Error Direction interaction was also significant, $\mathbf{F}(4,36)=7.00, \mathrm{p}<.001$. This was largely due to the fact that, although there was an overall trend for negative error performance to be better than positive error performance, there was an inversion at the 50 proportion.

\section{EXPERIMENT 2}

Seven U.S. Army enlisted men assigned to HumRRO, Division No. 5 , Fort Bliss, Texas, served as Ss.

The apparatus was identical to that used in Experiment 1, except that 154 slides were used, including 34 practice slides.

The procedure was similar to that used in Experiment 1. During the practice slides and during the presentation slides, Ss were instructed to respond "yes" if the number in the box agreed with their proportion estimate of the background or "no" if the number in the box did not agree. Ss responded to 120 presentation slides, which were presented in three blocks of 40 slides. Each slide was presented for $5 \mathrm{sec}$, and then $5 \mathrm{sec}$ were allowed for the recording of responses.

A variation of the method of constant stimuli, described by Dember (1965) and using a yes-no response, was employed. Normally, when this method is employed a constant standard is utilized, as was done in Experiment 1 when determining the five separate differential thresholds. However, in Experiment 2 the stimuli were constructed by randorn assignment of proportions between 20 and 80 to combinations of error direction and error size. This random assignment of proportions was done to investigate performance generalized over this range of proportions. The data were used to calculate error detection percentages and to compare detection performance with different error sizes and error directions, employing a 5 by 2 repeated measures design with seven $S$, with the dependent measure being the number of correct responses. This design involved 5 different error sizes $(3,6,9$, 12 , and 15) and 2 error directions (positive, negative).

The percentages of correct responses with regard to error size and error direction are presented in Table 2. A significant main effect for error size was observed, $F(4,24)=$ 18.67, $\mathrm{p}<.001$. A Duncan's test indicated that performance at each of the five levels of error size was significantly different from all other levels. The error direction effect was significant, $F(1,6)=4.80, p<.10$. Ss responded correctly to $60 \%$ of the positive errors and $72 \%$ of the negative errors.

\section{DISCUSSION}

On the basis of studies by Shuford (1961) and Stevens \& Galanter (1957) involving proportion estimation, it had been hypothesized in Experiment 1 that a plot of obtained differential thresholds would roughly approximate a binomial distribution with the mean around the 50 proportion. Shuford (1961) suggested that in proportion estimation tasks involving two types of objects, i.e., the circled and uncircled + signs in the present study, where the proportion of one object type is being estimated, a type of switching occurs at about the $50 \%$ point on the proportion continuum. This means that the other type of object is actually being estimated above the $50 \%$ point. For example, if two objects were the circled and uncircled + signs, when the proportion of circled + signs exceeded $50 \%$ the Ss would actually estimate the percentage of uncircled + signs and subtract it from $100 \%$ to give the percentage of circled + signs. If this reversal or switching is characteristic of proportion estimation tasks, then binomial performance plots would be expected. These binomial distributions would involve a mirror of performance on either side of the $50 \%$ point. The differential thresholds obtained in the present study (see Table 1) did not suggest a binomial distribution. They indicated that the task became progressively more difficult up to the $50 \%$ point, then leveled off. This leveling off suggested that no appreciable switching occurred. It is reasonable to assume that the nature of the experimental task, because it involved more than simple proportion estimations, may have prevented any appreciable switching from occurring.

In Experiment 2 the main effect of error size appeared approximately linear (see Table 2). The results, which indicated that performance at each of the error size levels was significantly different from all the remaining levels, appeared to contradict the results from Experiment 1, in which there was no significant difference in performance between error sizes of 10 and 15 . Quite possibly, the differences in the experimental tasks and the different error sizes employed may have been the chief contributors to this discrepancy in results.

It is interesting to note in

Table 2

Percentage of Correct Responses from Experiment 2

\begin{tabular}{cccc} 
& \multicolumn{3}{c}{ Direction } \\
\cline { 2 - 4 } Error & + & - & Mean \\
\hline 3 & 43 & 44 & 44 \\
6 & 47 & 61 & 54 \\
9 & 54 & 77 & 66 \\
12 & 68 & 88 & 78 \\
15 & 87 & 87 & 87 \\
Mean & 60 & 72 &
\end{tabular}


Experiment 2 that Ss performed significantly better in detecting negative errors than in detecting positive errors. Although the error direction main effect in Experiment 1 was not significant, there was a trend for negative error performance to be better than positive error performance, with the exception of an inversion at the 50 proportion. It appears that $\mathrm{Ss}$ tended to be more sensitive to negative discrepancies than to positive discrepancies. This perceptual tendency warrants further investigation.

It should be noted that the present study was intended to provide some "ballpark" data. It would be profitable in future research to employ more Ss and to require more judgments per stimulus. If more judgments were obtained, it would allow the calculation of DLs for each individual $\mathrm{S}$ which could be combined for the group. It is also conceivable that the particular selection of stimuli may have a marked effect on the results. Perhaps the results would have been different if instead of circling the + signs two different symbols had been used, such as the horizontal and vertical bars used by Shuford (1961).
Future research can explore this possibility.

\section{REFERENCES}

CORSO, J. F. The experimenial psychology of sensory behavior. New York: Holt, Rinehart \& Winston, 1967

DEMBER, W. N. The psychology of perception. New York: Holt, Rinehart \& Winston, 1965

SHUFORD, E. H. Percentage estimation as a function of element type, exposure time. and task. Journal of Experimental Psychology, 1961, 61, 430-436.

STEVENS, S. S., \& GALANTER, E. H. Ratio scales and category scales for a dozen perceptual continua. Joumal of Experimental Psychology, 1957, 54, 377-411. 